\title{
tic\&société
}

Vol. 12, $\mathrm{N}^{\circ} 1$ | 1er semestre 2018

Communs numériques et communs de la connaissance

\section{La culture comme commun : une approche à préciser}

Valérian GUILLIER

\section{(2) OpenEdition}

Journals

Édition électronique

URL : http://journals.openedition.org/ticetsociete/2350

DOI : 10.4000/ticetsociete. 2350

Éditeur

Association ARTIC

Édition imprimée

Pagination : 43-68

Référence électronique

Valérian GUILLIER, «La culture comme commun : une approche à préciser », tic\&société [En ligne], Vol. 12, $\mathrm{N}^{\circ} 1$ | 1er semestre 2018, mis en ligne le 31 mai 2018, consulté le 10 octobre 2020. URL : http:// journals.openedition.org/ticetsociete/2350 ; DOI : https://doi.org/10.4000/ticetsociete.2350 
tic\&société - 12(01), 2018

\title{
La culture comme commun : une approche à préciser
}

\author{
Valérian GUILLIER
}

Valérian Guillier est doctorant au sein du laboratoire Scènes du monde, création, savoirs critiques de l'Université de Paris 8 . II développe ses recherches sur la culture comme commun, en interrogeant le rapport aux outils numériques et d'Internet dans les formes d'auto-organisation collective. Son travail de thèse se nourrit de la sociologie et de l'économie de la culture et des NTIC. Valérian Guillier bénéficie d'un contrat doctoral par le Labex Arts$\mathrm{H} 2 \mathrm{H}$. valerian.guillier@etud.univ-paris8.fr 


\section{Valérian GUILLIER}

\section{La culture comme commun : une approche à préciser}

Résumé : Les communs culturels ont été utilisés pour décrire un large éventail de pratiques qui correspondent au sens anglo-saxon du terme culture. Ils ont aussi été utilisés pour comprendre les transformations dans la production et la réception des biens symboliques, notamment du fait des ordinateurs connectés en réseau. Dans cet article, nous étudions la possibilité d'analyser les biens symboliques comme des communs et comparons des définitions et usages du terme de communs appliqués aux biens symboliques. Dans le déroulement de l'article, la définition passe de l'accès ouvert - biens dans le domaine public ou du fait des licences libres -, considéré comme des Common Pool Resources (ressources en propriété partagées), à une définition prenant plus en compte l'importance de la communauté et de l'utopie politique dans les communs.

Mots-clés: communs, communs culturels, biens symboliques, accès libre, licences libres.

Abstract: Cultural commons have been used to describe a very large number of practices corresponding to an Anglo-Saxon understanding of culture. They have also been used in order to describe the changes in production and reception of symbolic goods, especially because of inter-networked computers. In this article, we explore the possibility of understanding symbolic goods as commons and compare different definitions and uses of the term commons applied to symbolic goods. Throughout the article, the definition ranges from open access-goods in the public domain or through free licenses-, considered as Common Pool Resources, to definitions highlighting the importance of community and political utopia in the Commons.

Keywords: commons, cultural commons, symbolic goods, open access, free licences. 
La culture comme commun : une approche à préciser

Resumen: Los comunes culturales han sido utilizados para describir un amplio conjunto de prácticas que se corresponden con el sentido anglosajón culture. Han sido también utilizados para comprender las transformaciones en la producción y en la recepción de los bienes simbólicos, especialmente como consecuencia de los ordenadores conectados en red. El presente artículo estudia la posibilidad de analizar los bienes simbólicos como comunes y se comparan las definiciones y usos de los comunes aplicados a los bienes simbólicos. A lo largo del artículo, la definición pasa del acceso abierto -bienes en el dominio público o como consecuencia de licencias libres-, considerado como Common Pool Resources (Recursos en propiedad compartida) a una definición que toma en cuenta la importancia de la comunidad y de la utopía política en los comunes.

Palabras clave: communes, communes culturales, bienes simbólicos, acceso libre, licencias libres. 


\section{Valérian GUILLIER}

Les communs permettent d'éclairer sous un jour nouveau certaines pratiques anciennes et de conceptualiser des évolutions, notamment dues aux transformations sociotechniques qui s'opèrent avec la généralisation des ordinateurs en réseau - au moins dans les pays du Nord. Envisager les productions culturelles comme communs ouvre un potentiel d'analyse riche pour différencier les pratiques de partage, de production en commun et les stratégies d'offre illimitée des nouvelles industries " créatives ». Mais alors quelle définition des communs conserver, dans un moment de foisonnement de la recherche sur le sujet ? La théorie des communs a permis d'analyser les communs naturels ou "fonciers » et, depuis le début des années 2000, de réaliser des analyses des communs informationnels, dans lesquels la culture a souvent été comprise. Pourtant, il semble nécessaire de préciser les termes. Celui de culture étant difficilement définissable, nous identifierons deux pôles autour desquels semblent graviter, dans la littérature, la plupart des acceptions des termes de culture comme "commun » ou de cultural commons. Nous nous concentrerons dès lors sur l'un de ces pôles, qui englobe la production, la diffusion et la réception des biens symboliques en montrant qu'ils sont des biens de la connaissance spécifiques et en tentant de circonscrire plus précisément ce que l'on peut entendre par "communs" dans les industries de ces biens particuliers.

Nous tacherons donc, dans cet article, de circonscrire les biens symboliques comme communs, d'abord selon la notion d'accès, pour intégrer par la suite celle de communauté et y adjoindre, enfin, ce qui nous semble être une nécessaire forme de revendication politique. Pour conclure, nous proposerons quelques exemples qu'il serait pertinent d'analyser comme des communs dans les domaines de la production, de la diffusion et de la réception de ces biens symboliques.

\subsection{Les communs et la culture}

Étudier les communs dans le domaine culturel nécessite de préciser les termes du débat. Les " communs culturels » (cultural 
La culture comme commun : une approche à préciser

commons) ont par exemple été mobilisés par Hess (2012) pour définir des pratiques collectives, traditionnelles, telle une compétition durant laquelle les participants faisaient rouler un fromage sur les pentes de Gloucester, en Angleterre. C'est également le sens proposé par Bertacchini, Bravo, Marrelli et Santagata (2012) quand ils affirment que « [I]es communs culturels [cultural commons] font référence aux cultures exprimées et partagées au sein d'une communauté ${ }^{1} »($ p. 3). Cette acception de la culture très utilisée dans les définitions anglo-saxonnes reste malgré tout large et complexe à caractériser.

De plus, les termes culture ou communs culturels seront évités, eu égard à la définition plus que complexe du terme culture. Dans leur tentative déjà ancienne de classification des différentes acceptions du mot, Kroeber et Kluckhohn (1952) recensent 164 définitions et plus qu'un concept difficile à circonscrire, ils envisagent le terme de culture comme :

une multitude de significations et de connotations qui peuvent être comparées à l'agglomération de la limaille de fer autour d'un aimant. Cette analogie peut être poussée plus avant: tout comme l'aimant est point de référence, les concepts clés sont au centre des cristallisations symboliques de chaque culture. Chargés d'affects, presque impossibles à délimiter et donc considérablement ouverts aux projections, ces concepts clés sont les références ultimes, conscientes et inconscientes, de la culture ${ }^{2}$ (p. 41).

Dans d'autres cas, comme chez Madison, Frischmann et Strandburg (2008), les communs culturels recouvrent même l'ensemble des communs informationnels, tel que le fait remarquer Coriat (2015). Or cette définition extensive du terme culture peut être mise en balance avec d'autres approches, comme celle d'Aigrain $(2005,2012)$, de Lessig (2004) ou de Berry et Moss

\footnotetext{
1 Traduction libre de : "Cultural commons" refer to cultures expressed and shared by a community. "

2 Traduction libre de : « magnet is a point of reference, so are the key concepts centers of symbolic crystallization in each culture. Charged with affect, almost impossible to delimit and hence susceptible to considerable projection, these fundamental concepts are the ultimate conscious and unconscious references in a culture. "
} 


\section{Valérian GUILLIER}

(2008). Ces auteurs semblent se concentrer sur ce qui, dans la culture, ne représente que l'ensemble des productions artistiques ou « créatives » d'une société. Le terme culture ne recouvre ici pas les pratiques collectives traditionnelles, mais se concentrent sur les biens symboliques, de leur création à leur réception.

Le terme culture, lorsqu'on envisage la culture comme commun, semble donc se situer entre deux pôles ainsi constitués :

- la culture au sens fréquemment utilisé par les AngloSaxons et l'ensemble des considérations ethnologiques et anthropologiques qu'elle recouvre ;

- la culture au sens entendu en France, lorsque nous abordons le domaine de la production, de la diffusion et de la réception des biens symboliques, tel qu'il est compris dans l'intitulé "Ministère de la Culture et de la Communication », ou dans les termes industrie(s) culturelle(s) - ce sont les communs culturels tendant vers ce second pôle que nous allons étudier par la suite.

Dans cet article, nous privilégierons les termes biens ou productions symboliques (utilisés de façon indifférenciée) pour caractériser les productions de l'esprit que nous souhaitons étudier. Nous reprenons le terme biens symboliques, notamment suite à Bouquillion, Miège et Mœglin (2013), qui proposent une analyse différenciée des industries culturelles et des industries créatives, tout en reconnaissant de nombreuses similitudes et influences réciproques. Les premières sont, selon les auteurs, des industries de biens reproductibles, qui doivent leur spécificité à la " coprésence qui y est ménagée en permanence entre exigence créatrice et contrainte reproductrice " (p. 16). Les industries créatives recoupent, selon eux, des industries de biens semireproductibles, qui veulent être assimilées à l'art et la culture pour leur dimension symbolique et pour leurs modes de fonctionnement (mise en valeur, distribution, financements). Le terme d'industries des biens symboliques se veut donc en ce sens recouvrir les deux industries sans les assimiler l'une à l'autre :

II nous semble plus pertinent, en l'occurrence, si l'on veut pouvoir juger des ressemblances et des écarts entre leurs modes 
La culture comme commun : une approche à préciser

respectifs de management créatif, d'en conserver la différence et de la marquer expressément en n'omettant pas non plus de rappeler que culture et créativité ne sont pas équivalentes. Mais par là - et moins contradictoirement qu'il n'y paraît - s'explique aussi le fait que nous tenions à conserver la dimension générique de ce que nous désignons par l'expression de "nouvelle économie des biens symboliques ", manière de conjuguer industries culturelles et industries créatives (Bouquillion, Miège et Mœglin, 2013, p. 10).

II s'agit également, pour les auteurs, d'éviter de participer à la légitimation de concepts attenants, qui brillent par le flou qui les caractérise, tels que " société créative » ou " économie créative ».

Le terme art sera aussi évité, car la qualification artistique ne relève pas de notre étude, qui se concentre sur les transformations des conditions de production et de réception des biens symboliques. La validation artistique est écartée du fait de l'objet étudié : les industries créatives relèvent de l'esthétisation d'objets industriels pourtant rarement artistiques; mais la validation artistique disparaît aussi dans certaines des nouvelles industries culturelles, notamment sur la plupart des plateformes commerciales ou non, où les vidéos, les textes, les photos ou les autres productions sont accumulés et valorisés sans distinction de qualité ou de qualification artistique. Les biens symboliques étudiés seront qualifiés d'« œuvres " dans les cas où nous les abordons dans leur dimension juridique. Là encore, il n'est pas question de la qualité puisqu'en droit, la protection s'applique « sur toutes les œuvres de l'esprit, quels qu'en soient le genre, la forme d'expression, le mérite ou la destination ${ }^{3}$ ".

Une fois ces précisions apportées, il convient de s'interroger sur l'opportunité d'appliquer les analyses du commun à une ressource aussi particulière que les biens symboliques.

\footnotetext{
${ }^{3}$ Article L-112-1 du Code le la propriété intellectuelle, France.
} 


\section{Valérian GUILLIER}

\section{Flou autour des biens symboliques comme communs}

Les productions symboliques entrent-elles dans le champ des communs de la connaissance ? II est certain que les productions symboliques sont au moins pour partie informationnelles. Un conte, un chant - traditionnel ou tube pop - peuvent être appréhendés comme de la connaissance, c'est-à-dire des mélodies, des textes, qui sont de l'information transmise (oralement ou par l'écriture). C'est l'objet des reprises et autres hommages que de réemployer au moins une part de l'information et d'en donner une autre interprétation. Pourtant, les biens symboliques ne sont pas réductibles à de l'information.

Les interprétations elles-mêmes sont porteuses de sens, et il suffit de regarder quelques-unes des milliers d'interprétations d'un personnage comme celui d'Hamlet pour se rendre compte de la variété des possibles et voir à quel point ils ne sont pas réductibles ni au texte original ni aux nombreuses incarnations passées. Audelà de la question de l'interprétation, les biens symboliques bénéficient de - ou génèrent - cette aura qu'évoque Benjamin (1936) lorsqu'il aborde la question de la reproduction mécanisée. Si les biens symboliques ne sont pas réductibles à leurs avatars physiques ou numériques, ils ne peuvent certainement pas se réduire à de la seule connaissance. La numérisation d'un tableau n'est pas l'équivalent de son original. Malgré tout, la dimension symbolique des productions naît de leurs circulations favorisées par les ordinateurs en réseau, mais plus largement dans les échanges (présentation à un public, intertextualité, etc.). La notion de divulgation, qui constitue un droit de l'auteur (parmi les droits moraux), révèle l'importance d'une rencontre avec le public pour la vie (ou la non-vie en cas de refus de divulgation ou de retrait) des productions symboliques - même si, en droit, l'œuvre est reconnue comme telle dès sa production, qu'elle soit divulguée ou non. Étant donné les spécificités étudiées, les biens symboliques ne sont pas exactement des biens de la connaissance traditionnels. II convient donc de se demander dans quelle mesure ils peuvent tout de même être appréhendés par la théorie des communs. 
La culture comme commun : une approche à préciser

Le terme de communs pour les biens symboliques ${ }^{4}$ a déjà été largement utilisé, mais rarement défini avec précision. Aigrain (2012) évoque par exemple tour à tour, dans son ouvrage Sharing, les communs culturels, numériques, culturels numériques, du partage, physiques, infrastructurels, les communs hors marché, sociaux et de la connaissance sans définir précisément ce qu'il entend par "communs ». Dans un ouvrage antérieur, il définit les «biens communs informationnels [comme] des créations qui appartiennent à tous parce qu'elles n'appartiennent à personne " (Aigrain, 2005, p. 31). Cette vision du commun est assez proche de celle défendue par Boyle (2003) dans sa lutte contre les enclosures 2.0. C'est une approche des communs par la question de l'accès. Boyle a en effet une vision du commun qu'il rapproche au domaine public, c'est-à-dire un espace de liberté et de gratuité permettant la créativité pour tous. Boyle inscrit sa définition des communs culturels à la croisée de celles de Lessig et de Benkler.

Lessig (2002) écrit : "Par commun, j'entends une ressource qui est gratuite. Pas nécessairement sans coût, mais s'il y a un coût, il est imposé de manière neutre, ou égale pour tous » (p. 180). Cette vision du commun est intimement liée au concept de culture libre (free culture) défini par la négative dans la préface du livre éponyme Free culture: How big media uses technology and the law to lock down culture and control creativity: "L'opposé de la culture libre est une "culture de la permission" [permission culture] - une culture dans laquelle le créateur ne peut créer qu'avec la permission des puissants, ou des créateurs du passé ${ }^{5}$ " (Lessig, 2004, p. xiv). La vision du domaine public de Benkler (1999) est encore plus fondée sur l'absence de barrière, c'est-à-dire que libre est entendu au sens où rien ni personne n'en entrave l'accès et l'utilisation. En somme, Boyle (2003) définit le commun entre Lessig, qui met l'accent sur la gratuité de l'accès à la ressource, et Benkler, qui met l'accent sur la liberté individuelle que permet une ressource dont l'utilisation n'impose aucune contrainte.

\footnotetext{
${ }^{4}$ Parfois englobés ou réduits par leurs auteurs aux termes de culture, d'art, voire de contenus.

${ }^{5}$ Traduction libre de : "The opposite of a free culture is a "permission culture"-a culture in which creators get to create only with the permission of the powerful, or of creators from the past. "
} 


\section{Valérian GUILLIER}

\subsection{Des ressources de propriétés partagées ?}

Quelle définition des communs conserver, qui permette l'étude des biens symboliques tout en s'inscrivant dans le mouvement des communs existants ? La première entrée en matière pourrait être celle de l'économie classique et de la vision naturaliste des modes de gestion des biens. La vision orthodoxe de l'économie classifie en effet les biens en fonction de la nature de ceux-ci. Selon cette doctrine, en posant la question de la rivalité et de l'exclusivité des expressions symboliques et culturelles, nous serions en mesure d'en déterminer la catégorie et, donc, nous disent les économistes, le modèle de gestion le plus approprié pour celles-ci (Tableau 1). C'est en partant de ce modèle qu'Ostrom élabore sa théorie des CPR (pour Common-pool resources, ou ressources de propriété partagée $\left.{ }^{6}\right)$. Ces ressources dont l'exclusion est difficile et pour lesquelles la rivalité est élevée sont celles autour desquelles la gestion des communs est la plus efficace. Pourtant, il convient de se demander si les productions symboliques sont des ressources rivales et non exclusives.

Tableau 1. Répartition des destinations par la nature des biens

\begin{tabular}{|l|l|l|}
\cline { 2 - 3 } \multicolumn{1}{c|}{} & Rivalité faible & Rivalité élevé \\
\hline Exclusion difficile & Biens publics & $\begin{array}{l}\text { Common-pool- } \\
\text { resources }\end{array}$ \\
\hline Exclusion facile & Biens privés & Biens de club \\
\hline
\end{tabular}

La rivalité est une question double dans le cas des biens symboliques. Si l'on considère l'artefact sur lequel se matérialisent les expressions symboliques et culturelles, il peut être rival ou non. Ainsi, un texte imprimé sera rival et m'en saisir en priverait

\footnotetext{
${ }^{6}$ Nous reprenons la traduction proposée par Valérie Peugeot dans l'ouvrage coordonné par l'association VECAM (2011) malgré l'absence regrettée dans la version française du terme commun.
} 
quelqu'un d'autre de l'usage. Cependant, si le même texte est sous un format numérique sur une machine connectée à Internet, celui-ci est alors non rival, car la lecture et la diffusion d'une copie de fichier n'empêchent pas l'utilisation du fichier d'origine, sauf en cas de présence d'un dispositif technologique ayant cette fonction restrictive ${ }^{7}$.

Un deuxième niveau de lecture en termes de rivalité des biens symboliques s'impose toutefois : avant d'être incarnées dans un artefact, fût-il numérique ou matériel, les expressions culturelles existent sous une forme non rivale. Madison, Frischmann et Strandburg (2008) envisagent cette " nature ${ }^{8}$ " des biens culturels qui englobent pour les auteurs les biens symboliques et l'ensemble de la connaissance :

En définitive, l'environnement intellectuel naturel consiste en une accumulation [vast pool] de ressources intellectuelles ouvertes au cœur desquelles et avec lesquelles nous vivons et nous lançons dans une grande variété d'activités et de pratiques $^{9}$ (p. 686).

Le même texte, s'il m'est lu, pourra m'être communiqué sans artefact et, avec le temps et de la mémoire, pourra m'avoir été intégralement partagé sans en avoir privé celui qui me l'a transmis. Je pourrai alors le réinterpréter - ce qui fait, par exemple, la richesse de la littérature orale. Cette propriété a pour conséquence de rendre l'exclusion des biens pour le moins complexe ${ }^{10}$. Un des exemples célèbres de cette non-rivalité " naturelle », qui a eu des

\footnotetext{
${ }^{7}$ Ces dispositifs appelés « mesures techniques de protection » (MTP), ou Digital Right Management (DRM,) sont omniprésents dans les produits commercialisés par les industries créatives, bien que peu efficaces car rapidement contournés après leur introduction.

${ }^{8}$ La naturalisation est aussi un phénomène qui touche la première dimension de la rivalité. En effet, il est fréquemment rappelé par les acteurs et les militants sur Internet que ce réseau est historiquement et intrinsèquement a-centré et que les ressources y sont naturellement non rivales, malgré les efforts actuels des différents acteurs dans la direction opposée.

9 Traduction libre de : "In sum, the natural intellectual environment consists of a vast pool of open intellectual resources within which and with which we experience life and engage in a wide variety of activities and practices. "

10 II n'est pas encore question, ici, de la circulation des biens hors du contrôle des industries créatives grâce aux ordinateurs en réseau.
} 


\section{Valérian GUILLIER}

effets sur l'« excluabilité » des biens symboliques, est le cas du Miserere composé par Allegri et tenu secret par le Vatican, que le jeune Mozart a pu retranscrire de mémoire. Si les éditions des avatars (ici les partitions) étaient limitées, donc permettaient l'exclusion, la représentation de l'œuvre en elle-même rendait possible sa duplication - à un esprit toutefois capable de saisir la complexité d'un chœur à six voix. Parlant des biens de la connaissance numériques, Hess (2011) nous explique que ceux-ci peuvent être appréhendés comme des ressources partagées qui avaient jusqu'ici les qualités de non-rivalité et de non-exclusivité propres aux biens publics. II s'agit donc collectivement d'en faire des CPR (ou de les envisager comme tels) et d'organiser la protection et la gestion des biens afin d'éviter qu'elles ne soient clôturées.

Hess propose en effet une définition ouverte des communs qui élargit les conceptions initiales d'Ostrom sur le sujet. Les biens symboliques ne peuvent être réduits à des biens publics comme le fait l'économie classique, dans la mesure où les catégorisations naturalistes des biens sont en fait dépassées par les problématiques rencontrées par les acteurs qui, dans leurs pratiques, luttent contre l'appropriation extérieure des biens en propriété partagée. Hess (2008) suggère que des glissements puissent se faire des biens publics vers les autres formes d'organisation des biens (privés, de club ou CPR). Ces glissements peuvent se faire par des modifications diverses (dans la loi, du fait de l'arrivée de nouvelles technologies, de nouveaux usages) et par la mise en place de communs, comme une des façons de conserver la gestion collective de la ressource. Cette conception du commun à protéger des enclosures est très similaire à celle de Boyle. On peut en effet envisager, avec les théoriciens américains du domaine public, que la constitution de biens symboliques est une activité nécessairement collective autour d'une ressource qui est ou doit rester publique. Selon Boyle, le processus amenant à de nouvelles productions de l'esprit nécessite un accès aux productions antérieures (d'être " assis sur les épaules du géant ", pour reprendre une expression attribuée à Newton). C'est le sens des limitations temporelles de la propriété intellectuelle, qui sont un équilibre entre l'intérêt de l'auteur et 
La culture comme commun : une approche à préciser

l'intérêt du public (pour avoir accès aux œuvres, mais aussi pour pouvoir en créer d'autres), ce que nous rappelle Boyle (2003) :

Le droit d'auteur [copyright] est en fait un système conçu pour nourrir le domaine public en accordant des droits limités et temporaires, eux-mêmes sujets à de considérables restrictions, même durant leur temps d'application - avec le but ultime de promouvoir le libre accès ${ }^{11}$ (p. 60).

Le domaine public peut alors être considéré comme un CPR, c'est-à-dire un ensemble de ressources dans lesquelles piocher pour en produire de nouvelles, qui finit par être en accès libre à tous, moyennant quelques ${ }^{12}$ années d'exceptions durant lesquelles l'auteur a le privilège de l'exclusivité dans l'exploitation. Une telle approche a mené les acteurs du libre accès à défendre une limitation des droits d'auteurs, de leurs durées et de leurs champs d'application. Ils ont aussi poussé en faveur de nouvelles exceptions, l'objectif avoué étant de limiter le champ des productions exclues temporairement du domaine public (pour des raisons telles que l'adaptation d'ouvrage aux personnes ayant un handicap, l'accès à la santé, l'accès à la connaissance et sa réutilisation à des fins pédagogiques, etc.). Si Boyle argumente en faveur d'une lecture du domaine public comme d'un commun, il n'envisage en réalité que la question de l'accès aux productions. Une fois cet accès garanti, libre et gratuit, Benkler (1999), pour sa part, considère l'ensemble des productions de l'esprit comme des communs. Pour autant, dans une définition plus proche de celle d'Ostrom et de Hess, la ressource ainsi constituée ressemble plus à un CPR, c'est-à-dire à des ressources autour desquelles peuvent s'organiser des communs bien plus qu'à des communs à proprement parler.

\footnotetext{
11 Traduction libre de " Copyright is, in fact, a system designed to feed the public domain providing temporary and narrowly limited rights, themselves subject to considerable restrictions even during their existence-all with the ultimate goal of promoting free access." "

${ }^{12}$ Aujourd'hui, en Europe et aux États-Unis, la période est tout de même de 70 ans après la mort de l'auteur.
} 


\section{Valérian GUILLIER}

\subsection{Le risque des enclosures}

Cette doctrine de l'accès s'est aussi incarnée hors du domaine public, notamment chez les partisans des licences libres appliquées à la création non logicielle. Celles-ci sont inspirées des licences libres appliquées au logiciel, notamment de la GNU GPL rédigée en 1989 pour formaliser et garantir juridiquement les revendications du mouvement du logiciel libre. Ces licences, dont les plus connues sont les licences Creative Commons, visent à formaliser un mouvement d'opposition à la culture commerciale similaire à l'opposition au logiciel propriétaire.

La vision des biens symboliques comme communs fondés sur l'accès libre et gratuit qui s'incarne dans ces licences a pour avantage de rendre évident un danger: les nouvelles enclosures, qui se matérialisent dans la prolifération des droits de propriété intellectuelle et dans l'élargissement, en droit comme en faits, de leur portée - au-delà des seules productions culturelles (recherche, savoirs traditionnels, etc.). Elle a aussi pour avantage d'être particulièrement bien adaptée aux conditions du numérique, dans lesquelles les ressources ne sont que rarement rivales et pour lesquelles l'enjeu se déplace de la sauvegarde d'un foncier fragile vers le développement, d'un intangible appropriable, donc pouvant être potentiellement soustrait du domaine public ou du domaine du libre (hors cas des licences copyleft ${ }^{13}$ ). La dimension protectrice du copyleft, qui tout en laissant la liberté à l'utilisateur final de faire ce qu'il veut des productions, oblige à ce que les œuvres dérivées ou composites produites restent dans le champ des productions libres et soumises à la même licence. Cette " viralité ${ }^{14}$ " est une bonne protection dans la doctrine de l'accès et une lutte contre les enclosures potentielles. Cependant, comme nous l'avons remarqué plus haut, elles constituent plus des CPR que des communs à proprement parler. Cette vision a donc le

\footnotetext{
13 Celles-ci sont tout de même sujettes à de la valorisation sans appropriation, notamment de la part des plateformes, qui valorisent l'attention du spectateur sans soucis de la propriété de la ressource, ce qui constitue le champ de recherche du Digital Labor.

${ }^{14}$ Le terme est déprécié par certains partisans du libre car connoté péjorativement mais revendiqué par d'autres comme un grain de sable dans les rouages d'une pratique d'appropriation systématique.
} 
La culture comme commun : une approche à préciser

désavantage de négliger, dans son analyse des communs, l'importance de l'action collective, de l'auto-organisation, c'est-àdire de la communauté, sinon pour considérer la spoliation qu'elle subit. Pourtant, la communauté construite autour des communs et ses modes d'auto-organisation sont des éléments centraux des pratiques et doivent être considérés y compris dans le cas des biens symboliques.

\section{De la culture libre au commun}

\subsection{Vers l'action collective}

Quelle définition du commun prend en compte l'importance de la production en commun, de la constitution de la communauté dans l'action de production et de gestion de la ressource ? Car le commun ne doit pas être réduit à une bonne gestion d'une ressource, dans la mesure où ce qui caractérise les communs informationnels et les biens symboliques a fortiori est qu'ils sont " construits" (Madison, Frischmann et Strandburg, 2008) : ils ne s'élaborent pas autour d'une ressource naturelle préexistante, mais autour de la production de la communauté. L'accès libre à une ressource ne semble pas suffisant pour définir un commun dans le domaine des biens symboliques - pour passer du CPR au commun. On perçoit ici que les combats autour des années 2000 ont accompagné, voire légitimé, une transition des modèles des industries créatives pour se diriger vers des services d'accès - le sens d' " accès " étant certes limité par rapport au sens qu'il a pris ci-dessus -, souvent illimités, aux catalogues de productions ${ }^{15}$. La logique de l'accès, la pensée d'un domaine public étendu par les licences ne permettent pas de constituer un commun, et une vision plus extensive des communs doit être mobilisée. Nous pouvons faire appel à la définition proposée par Coriat (2015), qui envisage les communs dans une articulation de trois éléments essentiels qu'il reprend à la lecture d'Ostrom et à la littérature existante :

\footnotetext{
${ }^{15}$ Des licences - dites à " réciprocité renforcée " - ont été conceptualisées même si aucune n'est réellement fonctionnelle. Elles envisagent toutes de dépasser ou de limiter cette appropriation rendue possible par les licences libres.
} 


\section{Valérian GUILLIER}

- une ressource mise en partage par une communauté ;

- un ensemble de règles pour organiser l'utilisation de la ressource ;

- un système de gouvernance, qui permet de faire évoluer démocratiquement les règles.

II s'agit donc d'envisager ce qui constitue une communauté et une gouvernance dans le domaine de la production et de la réception des biens symboliques. De prime abord, on pourrait envisager les plateformes sur lesquelles s'échangent la plupart des productions sur Internet comme créatrices de communautés tel que le suggère le vocabulaire commercial des plateformes ${ }^{16}$. YouTube, Flickr ou autre Wattpad sont en effet des plateformes ou des biens symboliques sont échangés, dans lesquelles des communautés se constituent, qui produisent parfois en commun, parfois en hommage, en réponse ou en citation d'autres productions et fréquemment en produisant des œuvres dérivées (mashup, fanfictions ou encore remix). Pourtant, la gouvernance de ces plateformes est entièrement centralisée par la société commerciale qui les détient et les changements parfois soudains de règles, ou leur application arbitraire, en sont des exemples criants. Quand YouTube décide de retirer une vidéo d'un vidéaste au motif qu'un robot a détecté une potentielle infraction au droit d'auteur - le robot détectant souvent de faux-positifs - mettant en péril la rémunération du vidéaste (qui parfois en dépend entièrement) et ne laissant que peu de recours (à l'issue irrémédiablement opaque), les membres de la "communauté " comprennent parfois dans la douleur qu'ils n'ont aucun pouvoir au sein de la plateforme. La question d'une gouvernance démocratique par les membres est donc primordiale et celle-ci ne s'engage pas autour de plateformes commerciales à but lucratif. Si

\footnotetext{
${ }^{16}$ Une manifestation récente est, par exemple, l'invitation de YouTube à rejoindre la communauté des YouTube Heroes au sein de laquelle vous pourrez avoir la joie de contribuer à améliorer le service et à faire de la modération en échange du droit à devenir bêta-testeur, autrement dit de produire de la valeur pour la plateforme pour gagner le droit d'en produire plus (YouTube Help, 2016). La vidéo originale a été supprimée depuis la rédaction de cet article, suite aux retours mécontents des utilisateurs et aux nombreuses parodies, mais elle reste accessible à https://archive.org/details/youtubeWh_1966valA.
} 
La culture comme commun : une approche à préciser

les plateformes constituent des communs infrastructurels, ce sont celles dont la gouvernance est exercée par ses membres qui devront retenir l'attention dans les recherches à venir sur les communs dans le domaine des biens symboliques.

Dans une autre direction, Rochfeld (2009) envisage que la communauté peut être pensée comme diffuse. L'auteure distingue cette catégorie d'une communauté "négative " (plutôt celle qui vaudrait pour le domaine public - ensemble de biens qui n'appartiennent à personne). Peut-être ces communautés diffuses peuvent-elles se constituer autour de contrats de licences ou d'autres obligations légales (Rochfeld prend l'exemple des monuments historiques) ? Elles ne s'organisent pas forcément de façon affirmée et ne sont que très peu définies, comme cela peut être le cas autour d'autres communs de la connaissance, la communauté constituée n'existant parfois pas ou ne représentant qu'une part minime des contributeurs. Dans le cas des productions symboliques sous licence libre, c'est même souvent une communauté diffuse en puissance qui est constituée. En effet, si l'on envisage qu'une production a le plus souvent un auteur, mais peut ensuite générer une ou plusieurs chaînes d'œuvres dérivées, permises et organisées par le contrat de licence libre, alors un bien symbolique sous licence libre peut générer une communauté diffuse résidant en puissance dans les conditions de la licence. D'autres productions sont immédiatement collectives, ce qui constitue une première communauté initiale, qui pourra par la suite être étendue si des œuvres dérivées sont produites.

Dans le cas d'une communauté diffuse - qui plus est en puissance -, la gouvernance doit être questionnée. En effet dans le cadre d'une licence, il n'est que difficilement possible de discuter les règles, puisqu'elles sont nécessairement "virales ". Quand il est possible de changer les règles, parce que la licence permet de le faire pour les œuvres dérivées, alors il ne s'agit toujours pas de délibérations collectives, mais d'un choix individuel de l'utilisateur qui change la licence, donc les règles qui s'appliquent à l'aval de sa chaîne de production. On pourrait alors parler de gouvernance partielle distribuée, au sens où chaque individu a le choix de travailler à partir des productions existantes (si le contrat de cession de droits d'auteurs l'autorise), mais doit alors accepter au 


\section{Valérian GUILLIER}

moins une partie des règles, qui ne sont jamais discutées collectivement. Chaque élément de la chaîne a un pouvoir sur l'aval de sa production sans pour autant avoir de pouvoir sur l'amont - sinon celui de ne pas produire d'œuvres dérivées s'il est en désaccord avec les règles en amont, car, contrairement aux exemples fréquemment donnés des logiciels libres très institués, dont le fonctionnement est régi par des organisations diverses et plus ou moins démocratiques, il est rare, voire inédit, que les biens symboliques soient organisés avec une gouvernance collective et démocratique.

Si l'on suit Coriat (2015), c'est à l'aune de l'effectivité des rules in use que s'apprécie la qualité de la structure de gouvernance. Ce serait donc à l'aune de l'effectivité des licences libres que s'évaluerait la gouvernance distribuée qu'elles engendrent. La dimension de communauté dans le cas de la production de biens symboliques reste néanmoins une notion complexe, tant la production est associée à un acte solitaire dans l'imaginaire collectif.

Évidemment, dans la production et la diffusion des biens symboliques, d'autres éléments entrent en compte en dehors des contenus eux-mêmes: c'est par exemple la mise en commun d'outils ou la création de communautés pour agir politiquement en faveur des communs dans le domaine des biens symboliques. Ainsi, comme le rappelle Paris (2011), reprenant Becker: " la valeur du produit se construit dans la production même des biens, mais aussi tout au long de la chaîne qui va amener le produit au consommateur. La valeur des biens symboliques n'est pas intrinsèquement contenue dans l'œuvre initiale, mais se sédimente au long de la chaîne des intermédiaires " (p. 58). II est donc nécessaire d'envisager l'ensemble des intermédiaires de production et de diffusion dans la réflexion sur les biens symboliques comme communs. Par ailleurs, il est loin d'être évident que les biens symboliques soient mis en partage dans les faits - et passent ainsi de CPR à communs effectifs -, y compris pour des raisons telles que la nécessité de vivre de son travail. En effet, l'adoption des licences est encore largement minoritaire, car 
La culture comme commun : une approche à préciser

la perspective d'une rémunération - qui s'avère très faible pour la plupart des sociétaires des sociétés d'auteurs ${ }^{17}$ - peut sembler compromise par une mise en partage effective des biens symboliques ${ }^{18}$.

Berry et Moss (2008) suggèrent une critique des licences libres en parlant d'un "commun sans communalité " (" a common without commonality »), remettant en cause la vision d'un commun pouvant se constituer sans communauté. II convient, comme nous l'avons déjà évoqué au sujet de la free culture et du domaine public, de distinguer les ressources disponibles des ressources partagées. Or les auteurs critiquent surtout la vision d'un commun qui ne se situerait pas par rapport au capitalisme dans un monde où le travail cognitif devient majoritaire dans la production et est largement exploité. II convient alors de revendiquer le commun comme antagonisme politique : considérer une ressource comme un commun peut déjà être compris comme une opposition à sa gestion par l'État ou le marché, un appel. La dimension principale du commun n'est pas, selon les auteurs, une composante «naturelle», mais une affirmation politique de la part de la communauté qui se constitue pour en assurer la gestion. Le passage du régime " naturel " au commun est un acte revendiqué et une définition des communs qui prend en compte la dimension politique et militante semble alors nécessaire.

\subsection{Vers la dimension politique}

Broca (2013) a montré, dans le monde du logiciel libre, que la pratique du développement logiciel est en réalité porteuse d'un éthos qui n'est pas nécessairement partagé par tous les acteurs du monde de l'open source, celui-ci étant focalisé sur l'efficacité, tandis que le libre est construit sur des valeurs morales qui s'incarnent dans les libertés accordées par la licence libre aux

\footnotetext{
17 L'analyse d'une répartition très inégalitaire débouchant sur un questionnement de l'optimum atteint a déjà été menée en 2015 (Langlais, 2015), mais pourrait être renouvelée en 2016 (SACD, 2017).

18 L'objet de cet article ne portant pas sur ce sujet, nous n'aurons pas l'occasion de développer ici de façon pertinente cette question, mais il est impossible d'évoquer les communs, notamment numériques et les biens symboliques, sans s'interroger sur les moyens de subsistance des contributeurs, qui restent une question sensible et cruciale.
} 


\section{Valérian GUILLIER}

utilisateurs. II convient alors de se demander si la pratique des communs relève aussi d'un éthos particulier autour des biens symboliques.

On peut en effet envisager la dimension politique des communs comme une de leurs constituantes importantes. Comme le logiciel libre affirme les libertés contre son pendant propriétaire, les biens symboliques comme communs s'opposent-ils aux industries culturelles ou créatives? Lessig (2005) dénonce une culture "read-only » imposée notamment par la propriété intellectuelle. II y oppose une culture " read-write ». Les deux termes (littéralement "lecture seule» et "lecture-écriture ») issus du monde informatique viennent proposer deux modèles de fonctionnement pour la culture sur les réseaux (Lessig, 2005). La définition du commun uniquement focalisée sur les règles de gestion ou sa dimension collective semble incomplète pour comprendre l'organisation des communs. II nous faut prendre en compte ce que Madison, Frischmann et Strandburg (2008) appellent un " récit " (« narrative ») pour les " communs culturels construits», qui doivent être, selon eux, analysés au même titre que les structures légales ou le contexte institutionnel. II s'agit en effet d'affirmer quelque chose en nommant "commun" une ressource: "Appeler quelque chose "commun de la connaissance" ou recaractériser certaines communautés de brevets comme des solutions aux problèmes des "anticommuns ${ }^{19 "}$ génère une attente tout à fait différente ${ }^{20} »(p .699)$.

C'est l'idée de Dardot et Laval (2014), qui entendent, dans leur ouvrage sur le commun, « identifier dans le principe politique du commun le sens des mouvements, des luttes et des discours qui, ces dernières années, se sont opposés à la rationalité néolibérale un peu partout dans le monde " (p. 18). Les auteurs refusent l'essentialisation du commun et affirment «que c'est seulement

\footnotetext{
${ }^{19}$ Les anticommuns ne sont pas détaillés ici, mais ils recouvrent les biens sur lesquels trop de personnes détiennent trop de droits entremêlés pour arriver à une gestion optimale de la ressource. Dans ce cas, les coûts de négociation sont trop hauts et, chacun exerçant ses droits à exclure, la ressource est sous-exploitée.

20 Traduction libre de "Calling something a "knowledge commons," or recharacterizing certain patent pools as solutions to "anticommons" problems, triggers a different set of expectations. »
} 
La culture comme commun : une approche à préciser

l'activité pratique des hommes qui peut rendre les choses communes, de même que c'est seulement cette activité pratique qui peut produire un nouveau sujet collectif » (p. 49 ; soulignement des auteurs). Ainsi, le commun est un mouvement qui permet d'unifier les combats, selon les auteurs, « un nouvel internationalisme » (p. 461), un nouvel élan dans la convergence des luttes.

Nicolas-Le Strat (2016) s'appuie sur ces travaux et ceux de Hardt et Negri pour proposer une définition intéressante du commun, dans la mesure où il essaie de réunir la nécessité d'une analyse fonctionnelle (l'étude des communs) avec une analyse d'un mouvement politique fondé sur ces pratiques d'autoorganisation (le commun) :

Un travail du commun opère donc un double déplacement: de la gestion étatique des affaires publiques vers une démocratie «du commun», impliquant un authentique espace public afin de délibérer entre les attentes et les préférences qui se font jour dans la société à ses niveaux les plus locaux et globaux avec, pour perspective, la formulation d'un intérêt commun (en matière de gestion de l'eau, de qualité de l'air, de politique de santé, d'urbanisation...) ; de la régulation arbitraire et inégalitaire des besoins par le marché vers une démocratie "des communs " (des biens et des services communs) avec là aussi la nécessité d'instituer un espace public de délibération en capacité d'évaluer les productions et créations qui se développent librement dans la société et se prononcer sur leur valeur aussi bien en termes d'utilité (en regard des droits humains à l'éducation, à l'alimentation, au soin, à la culture, au logement...) que d'émancipation (en regard du droit politique à la liberté, à l'égalité...) (p. 29).

Ainsi, il s'agit aussi d'envisager les biens symboliques comme un commun, dans leur acception politique : quel est l'engagement lorsque l'on envisage la production et la diffusion des biens symboliques comme un commun ? Cette ambition politique est-elle réalisée? 


\section{Valérian GUILLIER}

\section{Conclusion : une ouverture pour continuer l'étude de la production et la réception des biens symboliques}

La possibilité d'envisager les biens symboliques, dans leur version numérique (intangible) ou dans les artefacts physiques, ouvre les potentialités d'étude des biens symboliques comme communs que nous ne mènerons pas dans cet article dont l'objet, plus modeste, était de chercher à déterminer si les biens symboliques pouvaient être considérés comme des communs et selon quelles définitions. Nous avons essayé de montrer que le commun doit être compris tout à la fois comme un mouvement collectif et une ambition politique, qui se nourrissent mutuellement, au-delà de la seule logique de l'accès. Une dernière étape consiste donc à identifier une première typologie de communs à étudier, au regard des définitions $d u$ commun, qui s'incarne dans et guide le développement des communs, lesquels sont donc des ressources mises en partage par une communauté, avec des règles d'usage et une gouvernance, même sommaires.

D'abord, la dimension la plus évidente est probablement l'ensemble des biens symboliques considérés par leurs producteurs comme des communs. On peut penser à des producteurs comme Nina Paley, qui revendique le libre au point d'y avoir consacré une partie conséquente de son travail, ou David Revoy, auteur de la bande dessinée Pepper\&Carrot, récemment au cœur des débats dans son milieu puisqu'éditée par Glénat sans toucher de droits d'auteurs, une pratique rendue possible par la licence libre Creative Commons BY choisie - l'éditeur contribue toutefois à un financement participatif récurrent mis en place par l'auteur.

Viennent ensuite les infrastructures nécessaires à la production et à la diffusion des biens symboliques qui peuvent être des communs, dans lesquels les producteurs de contenus sont impliqués dans la gouvernance. Des projets existent et préexistaient à Internet, ou se sont construits sur des fondations plus anciennes qu'Internet; ainsi, CD1D, une plateforme réunissant des labels indépendants, propose un site Internet permettant de télécharger de la musique ou de l'écouter de façon 
La culture comme commun : une approche à préciser

" équitable ». D'autres infrastructures plus complexes mais nécessaires au commun se mettent en place. C'est le cas de la C3S, alternative en construction à la GEMA ${ }^{21}$, la société de collecte de droits pour les auteurs de musique en Allemagne. Cette nouvelle société d'auteur vise notamment les producteurs de contenus sous licence libre et a d'ailleurs un manifeste intitulé $L e$ manifeste pour le commun culturel (Das Cultural Commons Manifest ; C3S, s. d.). II convient alors d'observer le fonctionnement des sociétés d'auteurs contre lesquelles un tel projet s'élabore pour voir si elles sont elles-mêmes des communs infrastructurels, l'ont été, et voir ce qui les différencie.

Enfin viennent d'autres organisations qui gravitent autour de la production de contenus et de leur diffusion et qui travaillent à faire reconnaître les biens symboliques ${ }^{22}$ comme communs. Ces organisations ne sont pas impliquées directement dans la production ou la diffusion, mais travaillent à l'analyse des textes de loi et à la rédaction d'éventuels amendements, à la rédaction de licences, à l'incitation à des pratiques ou à l'organisation d'évènements qui développent l'idée que les biens symboliques sont ou devraient être des communs. II s'agit de collectifs comme SavoirsCom1, Museomix, Remix The Commons ou même une organisation comme Creative Commons, qui valorise les contenus sous licence libre, fait du lobbying et met en place un espace de discussion pour les évolutions futures des licences. D'autres collectifs, comme celui construit autour de la liste Copyleft attitude et de la licence Art Libre organisent des rencontres régulières, pour le plaisir de la rencontre.

Ces trois types de communs potentiels participent de la transformation des conditions de production et de réception des biens symboliques. II s'agira dès lors, par l'étude de cas, de comprendre dans quelle mesure se construisent des communs réels et dans quelle mesure elles participent d'un éthos partagé.

\footnotetext{
${ }^{21}$ Die Gesellschaft für musikalische Aufführungs- und mechanische Vervielfältigungsrechte.

22 Ces organisations parlent parfois de culture, ou des arts voire de la connaissance comme communs.
} 


\section{Valérian GUILLIER}

\section{Références}

Aigrain, P. (2005). Cause commune: l'information entre bien commun et propriété. Paris, France : Fayard.

Aigrain, P. (2012). Sharing: Culture and the Economy in the Internet Age. Amsterdam, Pays-Bas: Amsterdam University Press.

Benjamin, W. (1936). L'œuvre d'art à l'époque de sa reproduction mécanisée. Zeitschrift für sozialforschung, 5(1), 40-58.

Benkler, Y. (1999). Free As the Air to Common Use: First Amendment Constraints on Enclosure of the Public Domain. New York university law review, 74(2), 354-446.

Berry, D. M. et Moss, G. (2008). Libre culture: Meditations on Free Culture. Winnipeg, Canada : Pygmalion Books.

Bertacchini, E., Bravo, G., Marrelli, M. et Santagata, W. (2012). Defining Cultural Commons. Dans E. Bertacchini, G. Bravo, M. Marrelli et W. Santagata (dir.), Cultural Commons (p. 3-18). Cheltenham, Angleterre : Edward Elgar Publishing.

Bouquillion, P., Miège, B. et Mœglin, P. (2013). L'industrialisation des biens symboliques: les industries créatives en regard des industries culturelles. Saint-Martin-d'Hères, France: Presses universitaires de Grenoble.

Boyle, J. (2003). The Second Enclosure Movement and the Construction of the Public Domain. SSRN Electronic Journal, 66, 33-74.

Broca, S. (2013). Utopie du logiciel libre : du bricolage informatique à la réinvention sociale. Neuvy-en-Champagne, France : Éditions Le Passager clandestin.

Code de la propriété intellectuelle, art. L112-1. (s. d.). Repéré à https://www.legifrance.gouv.fr/affichCode.do?idSectionTA=LEGI SCTA000006161634\&cidTexte $=$ LEGITEXT000006069414\&date Texte $=20180319$

Coriat, B. (dir.). (2015). Le retour des communs: la crise de l'idéologie propriétaire. Paris, France: Éditions les Liens qui libèrent. 
La culture comme commun : une approche à préciser

Dardot, P. et Laval, C. (2014). Commun : essai sur la révolution au $X X l^{e}$ siècle. Paris, France : La Découverte.

Cultural Commons Collecting Society (C3S) (s. d.). Das CulturalCommons-Manifest. Récupérée à https://www.c3s.cc/ueberc3s/manifest/

Hess, C. (2008). Mapping the New Commons. Présenté à Governing Shared Resources: Connecting Local Experience to Global Challenges; the $12^{\text {th }}$ Biennial Conference of the International Association for the Study of the Commons, University of Gloucestershire, Cheltenham, Northampton, MA. https://doi.org/10.2139/ssrn.1356835

Hess, C. (2011). Inscrire les communs de la connaissance dans les priorités de recherche. Dans V. Peugeot (dir.), Libres savoirs: les biens communs de la connaissance: produire collectivement, partager et diffuser les connaissances au $X X I^{e}$ siècle (p. 33-54). Caen, France : C\&F éditions.

Hess, C. (2012). Constructing a New Research Agenda for Cultural Commons. Dans E. Bertacchini (dir.), Cultural Commons: A New Perspective on the Production and Evolution of Cultures (p. 19-35). Cheltenham, Angleterre/Northampton, MA : Edward Elgar.

Kroeber, A. L. et Kluckhohn, C. (1952). Culture: A Critical Review of Concepts and Definitions. Cambridge, MA : The Museum.

Langlais, P.-C. (2015, 8 avril). Le droit d'auteur ne fait vivre qu'une infime minorité d'artistes. L'OBS avec Rue89. Récupérée à https://www.nouvelobs.com/rue89/rue89-rue89-

culture/20150408.RUE8597/le-droit-d-auteur-ne-fait-vivre-quune-infime-minorite-d-artistes.html

Lessig, L. (2002). The Architecture of Innovation. Duke Law Journal, 51(6), 1783-1801.

Lessig, L. (2004). Free Culture: How Big Media Uses Technology and the Law to Lock Down Culture and Control Creativity. New York, NY : Penguin Press. 


\section{Valérian GUILLIER}

Lessig, L. (2005, 28 décembre). Creatives Face a Closed Net. Financial Times. Repéré à https://www.ft.com/content/d55dfe52-77d2-11da-9670$0000779 \mathrm{e} 2340$

Madison, M. J., Frischmann, B. M. et Strandburg, K. J. (2008). Constructing Commons in the Cultural Environment. Legal Studies Research Paper Series, 95, 657-710.

Nicolas-Le Strat, P. (2016). Le travail du commun. Saint-Germainsur-Ille, France : Éditions du commun.

Paris, T. (2011). Des industries culturelles aux industries créatives: un changement de paradigme salutaire?. tic\&société, 4(2), 42-54.

Rochfeld, J. (2009). Entre propriété et accès: la résurgence du commun. Dans C. Noiville et F. Bellivier (dir.), La bioéquité : batailles autour du partage du vivant (p. 69-88). Paris, France : Éditions Autrement.

Société des auteurs et compositeurs dramatiques (SACD). (2017). Rapport annuel exercice 2016. Si ce n'est pour la culture "pourquoi nous battons-nous alors?". Repéré à https://www.sacd.fr/sites/default/files/rapport_annuel_2016.pdf

VECAM. (dir.). (2011). Libres savoirs: les biens communs de la connaissance : produire collectivement, partager et diffuser les connaissances au $X X l^{e}$ siècle. Caen, France : C\&F éditions.

YouTube Help. (2016). Getting Started with YouTube Heroes. Repéré à https://archive.org/details/youtube-Wh_1966valA 